\title{
The influence of age and dosage on the pharmacodynamics of dexmedetomidine in rabbits
}

\author{
Agnieszka Bienert', Włodzimierz Płotek², Paweł Wiczling ${ }^{3}$, Justyna Warzybok', \\ Katarzyna Borowska ${ }^{7}$, Katarzyna Buda ${ }^{4}$, Karolina Kulińska ${ }^{4}$, Hanna Billert ${ }^{4}$, \\ Roman Kaliszan³, Edmund Grześkowiak ${ }^{1}$ \\ ${ }^{1}$ Department of Clinical Pharmacy and Biopharmacy, Poznan University of Medical Sciences, Poland \\ 2 Department of Teaching Anaesthesiology and Intensive Therapy, Poznan University of Medical Sciences, Poland \\ ${ }^{3}$ Department of Biopharmaceutics and Pharmacodynamics, Medical University of Gdańsk, Poland \\ ${ }^{4}$ Department of Experimental Anaesthesiology, Poznan University of Medical Sciences, Poland
}

\begin{abstract}
Aim. This study aimed to examine the influence of maturation and dosage on the sedative and haemodynamic response observed in rabbits after the administration of dexmedetomidine.

Material and methods. The pharmacodynamics of dexmedetomidine was studied on 14 healthy New Zealand white rabbits at three periods of maturation; stage 1-1.5 months old, stage 2-2.5 months old and stage 3-6.5 months old ones. The administered dose of dexmedetomidine ranged from $25 \mu \mathrm{g} / \mathrm{kg}$ to $300 \mu \mathrm{g} / \mathrm{kg}$ of body weight. The pedal withdrawal reflex was used to measure the duration of anaesthesia. The heart rate and mean arterial pressure were measured at the third stage of the study to evaluate the haemodynamic response. A simple pharmacodynamic relationship between the dose and the duration of anesthesia was used to describe the data. Results. We observed that young rabbits were less sensitive to dexmedetomidine than adult animals, as was reflected by the pedal withdrawal reflex, and we found that the haemodynamic response to dexmedetomidine depended on dosage of the drug. Dexmedetomidine decreased the mean blood pressure in a dosage-dependent manner with the highest decrease observed for the lowest dose. As the dose increased, the hypotensive effect of the drug was less noticeable. After the administration of dexmedetomidine the heart rate decreased to the same value regardless of the dose applied.
\end{abstract}

Key words: dexmedetomidine, rabbits, pedal withdrawal reflex.

\section{Introduction}

Dexmedetomidine is an $\alpha 2$-agonist used for a shortterm sedation of adults in the ICU. The central hypnotic mechanism is not associated with GABA, as in the case of benzodiazepines and propofol. The stimulation of an adrenergic system in the central nervous system at locus coeruleus elicits a conscious sedation whereas the analgesic properties are the effect of substance $P$ suppression in the dorsal horn of the spinal column. Cardiovascular effects are bradycardia and hypoten- sion. The lack of respiratory inhibition is one of the advantages of the drug [1]. Dexmedetomidine exerts cardioprotective, nephroprotective, hepatoprotective and neuroprotective activity, and the latter one seems to be of special importance to patients in the ICU, probably preventing the occurrence of delirium $[2,3]$.

Clinical studies on healthy volunteers have shown that dexmedetomidine pharmacokinetics is best described by a two-compartment model. The distribution phase is rapid with a half-life of $6 \mathrm{~min}$. The drug 
is highly protein bound. The volume of distribution at steady state equals $1.33 \mathrm{~L} / \mathrm{kg}$ or $118 \mathrm{~L}$. Metabolism of dexmedetomidine occurs mainly in the liver by cytochrome P450 (CYP) enzyme 2A6 and uridine diphosphate glucuronosyltransferase (UGTs) glucuronidation pathways, specifically UGT1A4 and UGT2B10, to inactive metabolites. Dexmedetomidine clearance equals $39 \mathrm{~L} / \mathrm{hr}$, and the elimination half-life of 2-2.5 hours [4].

Currently, dexmedetomidine in humans has been registered for sedation. There are still intensive scientific studies on possibilities of dexmedetomidine perioperative application in anaesthetized patients as premedication, adjuvant in neuraxial and general anaesthesia. The scientists test its narcotic-sparing effects, as well as effectiveness for awake fiberoptic intubation, and intraoperative sedation [2].

Dexmedetomidine, despite of the fact that it is registered only for adults, has been recently proposed for sedation in the pediatric ICU settings [5]. However, the problem which occurs in almost every drug administered in the PICU settings, is the lack of detailed pharmacokinetic and pharmacodynamic information on the drug in children population. Up to $70 \%$ of the drugs in pediatric intensive care and $90 \%$ of the drugs in neonatal intensive care, are prescribed in an off-label or unlicensed manner. Pediatric dosing regimens are usually empirically derived from adult regimens using linear extrapolations based on body weight. However, this calculation does not take into account all the physiological changes occurring during the maturation process. Child-ren differ from adults in their response to drugs, one of the reason may be a different metabolism which is faster in children than in adults. These differences cause changes in the pharmacokinetics (PK) and/or pharmacodynamics (PD) of drugs and they may also vary among children of different ages. This may lead to therapeutic failure and severe adverse effects or even death, like in the example of fatal complication after propofol infusion used for long-term sedation in neonates $[6,7]$. Large interindividual differences in the pediatric population and also the need to broaden patients group to children for most of commonly used drugs show the problem which is occurring in PICU settings. The lack of PK and PD information on drugs in children has led to the European Regulation, which came into force in 2007. This law recommends to perform the studies in children in the early stages of the development of a new drug $[6,8,9,10]$. Due to the ethical reason, it is difficult to eliminate the problem of interindividual variability and examine the influence of maturation process on pharmacodynamics of a given drug in one child in different stages of its ripeness. The study conducted in the animals seems to be a rational solution.

To examine pharmacodynamic response to given drug in the laboratory setting, scientists use various reflexes of different animals. One of them is the pedal withdrawal reflex. This reflex was proposed for assessing the sedative pharmacodynamic response to various drugs: propofol [11], midazolam [12], diazepam-ketamine-pentazocine [13] in rabbits. The aim of our study was to examine the influence of the maturation process on the sedative pharmacological response to dexmedetomidine in rabbits and to determine the influence of different doses of dexmedetomidine on rabbits' monitored hemodynamic parameters.

\section{Materials and methods}

All experiments with animals were conducted with approval by the local animal care committee and their care was in accordance with institutional and international guidelines.

\section{Animals}

The study was divided into 3 periods according to three stages of animals' growth. 14 healthy New Zealand white rabbits with the average weight of $1.2 \pm 0.1 \mathrm{~kg}$ (mean \pm SD), aged 42-54 days, were used in the first period. In the second period the average weight and age of the animals were $1.8 \pm 0.2 \mathrm{~kg}$ and $75-85$ days, respectively, whereas in the third period they were $3.2 \pm 0.4 \mathrm{~kg}$ and 169-214 days, respectively. Two rabbits were not used in the second period due to the procedural reasons, whereas three new adult animals were added during the third period to examine the haemodynamic response to dexmedetomidine.

All the rabbits were housed individually in stainless steel cages under controlled environmental conditions. The room temperature and relative humidity were controlled at $20-22^{\circ} \mathrm{C}$ and $50-60 \%$, respectively. The rabbits were provided with $125 \mathrm{~g}$ of commercial pelleted diet once a day between 08:00 and 12:00 $\mathrm{h}$ and they drank tap water ad libitum. Dexmedetomidine (Dexdomitor $0.5 \mathrm{mg} / \mathrm{ml}$, Orion Corporation, Finland) was administered intravenously as a single bolus at different doses $(25 \mu \mathrm{g} / \mathrm{kg}, 35 \mu \mathrm{g} / \mathrm{kg}, 50 \mu \mathrm{g} / \mathrm{kg}, 75 \mu \mathrm{g} / \mathrm{kg}$, $100 \mu \mathrm{g} / \mathrm{kg}, 140 \mu \mathrm{g} / \mathrm{kg}, 150 \mu \mathrm{g} / \mathrm{kg}, 200 \mu \mathrm{g} / \mathrm{kg}, 250$ $\mu \mathrm{g} / \mathrm{kg}, 300 \mu \mathrm{g} / \mathrm{kg}$ of body weight). The experiment was conducted from November, 2013 till April, 2014.

Animals were fasted on the day of sedation. Just before infusion, the rabbits were weighed and placed into restraining cages. The hair over the auricular artery 
and on the tail was removed and the skin cleaned with alcohol. A 22G catheter was inserted percutaneously into the central auricular artery and fixed with tape. Dexmedetomidine was administered to the marginal vein of the opposite ear. Warm fluids $\left(38^{\circ} \mathrm{C}\right)$ were infused after each blood sampling. Rabbits from period 3 of examination additionally had arterial catheter attached to monitoring system Philips IntelliVue MP5 with Philips M1567A catheter and sedation included monitoring of cardiac and respiratory status.

The animals were oxygenated with 100\% oxygen at $3 \mathrm{~L} / \mathrm{min}$ via a facial mask; oxygen flow was continued until the animals recovered completely. Appropriate dose of dexmedetomidine was administered as a bolus injection. Rabbits from stage 3 of examination had additionally monitored heart rate and recorded from the curve of the arterial blood pressure during the first 30 minutes from administration. Blood oxygen saturation was monitored from the shaved tail by pulse-oximetry.

In order to monitor the level of sedation, two basic reflexes, i.e. pedal withdrawal reflex and corneal reflex, were tested. The reflex was tested in the following periods: initially, every 20,40, 50 and 60 seconds, and every few minutes after administration, until full recovery. In this study, the recorded starting point and endpoint was loss and return of the pedal withdrawal reflex, whereas the corneal reflex was always retained during the experiments. The reflex is elicited by extending the limb and stimulating it to achieve its withdrawal.

\section{Pharmacodynamics}

The main parameter derived from pedal withdrawal reflex measurements is the duration of anesthesia $\left(t_{d}\right)$. Assuming an IV infusion and one compartment disposition model the duration of anesthesia can be described by the following equation (1) [14]:

$$
t_{d}=\frac{1}{k}\left(\ln D-\ln D_{\min }\right) \longrightarrow
$$

where $k$ denotes elimination rate constant, $D$ denotes the administered dose, and $D_{\min }$ denotes the minimum effective dose. For more complex PK the equation can also be used, as the terminal (the slowest) phase will mostly contribute to the time of responsiveness after administration of an anaesthethic. In such case, $k$ will denote the slope of the terminal phase.

The statistical analysis was done in Matlab ${ }^{\circledR}$ Software version 7.0 (The MathWorks, Inc., Natick, MA, USA) using the Curve Fitting Tool.

\section{Results}

Table 1 shows comparison between administered dose of dexmedetomidine and rabbit response on the basis of loss and return of pedal withdrawal reflex. In all stages of our research, time required for loss and return of the reflex shortens and elongates, respectively, with given dose of dexmedetomidine. Small doses entail more time to cause anaesthesia and consequently shorten the duration of sedation. The higher doses of dexmedetomidine the longer is sedation and more time needed for pedal withdrawal reflex return.

The proposed model adequately described the experimentally determined durations of anesthesia (td) as shown in Figure 1. The linearity of anaesthesia duration with respect to logarithm of dose indicated that a linear PK can be expected within the studied range of doses. The following parameter estimates (coefficient of variation) were obtained for Stage 1: $D_{\min }=20.8(28.5 \%)$ $\mathrm{mg} / \mathrm{kg}$ and $\mathrm{k}=0.0369(17 \%) \mathrm{min}^{-1}$; Stage $2: D_{\min }=11.4$ $(39.8 \%) \mathrm{mg} / \mathrm{kg}$ and $k=0.0411(18.8 \%) \mathrm{min}^{-1}$; and Stage 3: $D_{\min }=8.60(83.0 \%) \mathrm{mg} / \mathrm{kg}$ and $k=0.0367$ (32.5\%) $\mathrm{min}^{-1}$. The obtained terminal elimination rate constants corresponded to half-lifes of $18.8 \mathrm{~min}$ (Stage 1), $16.9 \mathrm{~min}$ (Stage 2) and 18.9 min (Stage 3). No age related changes of terminal elimination rate constant were observed. However, a decrease of a minimal effective dose with rabbits age was evident. It suggested that young rabbits were less sensitive for dexmedetomidine than old ones.

Influence of the drug on blood pressure and heart rate was summarized in Table 2 and Figure 2. The baseline MAP equaled $92 \pm 8.4 \mathrm{mmHg}$. It decreased on average upon dexmedetomidine administration to the value of $76 \pm 9.6 \mathrm{mmHg}$. Interestingly, the highest decrease was observed for the smallest dose $(35 \mu \mathrm{g} / \mathrm{kg})$. For larger doses the decrease in MAP was less apparent. The significant slope of $0.103 \mathrm{mmHg} /(\mathrm{mg} / \mathrm{kg})$ between mean MAP and dexmedetomidine dose was observed. This value indicates that the increase in the dexmedetomidine dose of $10 \mu \mathrm{g} / \mathrm{kg}$ is followed by the increase in mean MAP of $10.3 \mathrm{mmHg}$. This equation seems valid for the range of studied doses $(35-250 \mu \mathrm{g} / \mathrm{kg})$. The dexmedetomidine also decreased the heart rate from the initial (baseline) values of $207 \pm 49.0$ beats $/ \mathrm{min}$ to $100 \pm 20.8$ beats $/ \mathrm{min}$ noted during the anesthesia. The decrease in heart rate was constant, independent of the administered dose.

\section{Discussion}

During the study we examined the relationship between the pharmacological response to dexmedetomidine and 
Table 1. Comparison of dose-dependent loss and return of pedal withdrawal reflex and duration of sedation in the subsequent stages of examination in healthy rabbits

\begin{tabular}{|c|c|c|c|c|}
\hline Dose & Rabbit no. & Loss of pedal withdrawal reflex & Return of pedal withdrawal reflex & Duration of sedation \\
\hline \multicolumn{5}{|c|}{ STAGE 1} \\
\hline$[\mu \mathrm{g} / \mathrm{kg}]$ & & [min] & {$[\mathrm{min}]$} & [min] \\
\hline 25 & 8 & 01:10 & $14: 30$ & $13: 20$ \\
\hline 35 & 11 & $02: 30$ & $15: 30$ & 13:00 \\
\hline \multirow{3}{*}{50} & 10 & $00: 35$ & 16:00 & $15: 25$ \\
\hline & 13 & $00: 40$ & 09:00 & 08:20 \\
\hline & 14 & $00: 20$ & 21:05 & $20: 45$ \\
\hline \multirow{2}{*}{75} & 6 & $00: 50$ & $36: 00$ & $35: 10$ \\
\hline & 9 & $00: 40$ & $35: 00$ & $34: 20$ \\
\hline 100 & 4 & 00:35 & $60: 00$ & $59: 25$ \\
\hline 140 & 3 & $00: 40$ & $59: 30$ & $58: 50$ \\
\hline 150 & 5 & $00: 20$ & $56: 00$ & $55: 40$ \\
\hline \multirow{2}{*}{200} & 1 & $00: 40$ & $27: 40$ & $27: 00$ \\
\hline & 7 & $00: 15$ & $67: 00$ & $66: 45$ \\
\hline 250 & 12 & 00:15 & 73:00 & $72: 45$ \\
\hline 300 & 2 & $00: 20$ & 80:00 & 79:40 \\
\hline \multicolumn{5}{|c|}{ STAGE 2} \\
\hline \multirow{2}{*}{35} & 8 & $00: 50$ & 17:00 & $16: 10$ \\
\hline & 11 & $00: 36$ & 19:00 & $18: 24$ \\
\hline \multirow{3}{*}{50} & 10 & $00: 20$ & $23: 30$ & 23:10 \\
\hline & 13 & $00: 25$ & $30: 20$ & 29:55 \\
\hline & 14 & $00: 35$ & 45:00 & $44: 25$ \\
\hline \multirow{2}{*}{75} & 6 & 00:55 & $57: 00$ & 56:05 \\
\hline & 9 & $00: 40$ & 59:00 & $58: 20$ \\
\hline 100 & 4 & $00: 30$ & $36: 00$ & $35: 30$ \\
\hline 150 & 5 & 00:50 & 59:16 & $58: 26$ \\
\hline \multirow{2}{*}{200} & 1 & $00: 30$ & $60: 00$ & 59:30 \\
\hline & 7 & $00: 26$ & $72: 00$ & 71:34 \\
\hline 250 & 2 & $00: 35$ & $80: 00$ & $79: 25$ \\
\hline \multicolumn{5}{|c|}{ STAGE 3} \\
\hline \multirow{2}{*}{35} & 8 & $01: 25$ & $27: 00$ & $25: 35$ \\
\hline & 11 & 01:15 & $35: 45$ & $34: 30$ \\
\hline \multirow{2}{*}{50} & 13 & 01:50 & $40: 00$ & $38: 10$ \\
\hline & 14 & $00: 50$ & $46: 00$ & $45: 10$ \\
\hline 75 & 6 & $01: 50$ & $55: 00$ & $53: 10$ \\
\hline \multirow{2}{*}{100} & 2 & 01:00 & $105: 00$ & $104: 00$ \\
\hline & 4 & 01:00 & $82: 00$ & $81: 00$ \\
\hline \multirow{2}{*}{150} & 17 & $00: 30$ & 73:00 & $72: 30$ \\
\hline & 18 & 00:50 & $95: 30$ & $94: 40$ \\
\hline \multirow{2}{*}{200} & 1 & $00: 45$ & $62: 16$ & $61: 31$ \\
\hline & 7 & 00:30 & 74:00 & 73:30 \\
\hline \multirow{2}{*}{250} & 16 & $00: 20$ & $127: 00$ & $126: 40$ \\
\hline & 19 & $00: 50$ & 53:00 & $52: 10$ \\
\hline
\end{tabular}

the process of maturation in rabbits and the influence of the dosage of dexmedetomidine on the haemodynamic parameters. There have been reports on the use of dexmedetomidine in other animals, like rats [15], dogs [16], cats [17] or gerbils [18], but only adult ones. The administration of dexmedetomidine has also been tested on adult rabbits, mainly in the context of neuroprotection, ventilatory and haemodynamic properties $[19,20]$. In our study we used the pedal withdrawal reflex to measure the duration of sedation after single intravenous administration. We found it useful for testing the level of sedation and anaesthesia both in adult 
Table 4. The baseline and average mean arterial pressure and heart rate in rabbits after dexmedetomidine administration at the $3^{\text {rd }}$ stage of the experiment

\begin{tabular}{|c|c|c|c|c|c|c|c|c|}
\hline \multirow{2}{*}{$\begin{array}{c}\text { Dose } \\
{[\mu \mathrm{g} / \mathrm{kg}]}\end{array}$} & \multirow[t]{2}{*}{ Rabbit no. } & \multirow{2}{*}{$\begin{array}{c}\text { Body weight } \\
{[\mathrm{kg}]}\end{array}$} & \multicolumn{3}{|c|}{ Mean arterial pressure (MAP) [mmHg] } & \multicolumn{3}{|c|}{ Heart rate (HR)bpm } \\
\hline & & & Baseline & Mean & Baseline- Mean & Baseline & Mean & Baseline- Mean \\
\hline \multirow{2}{*}{35} & 8 & 3.2 & 105 & 68 & 37 & 230 & 128 & 102 \\
\hline & 11 & 3.1 & 86 & 63 & 23 & 235 & 100 & 135 \\
\hline \multirow{2}{*}{50} & 13 & 2,9 & 84 & 62 & 22 & 129 & 84 & 45 \\
\hline & 14 & 2.8 & 94 & 64 & 30 & 190 & 159 & 31 \\
\hline \multirow{2}{*}{75} & 6 & 3.3 & 95 & 70 & 25 & 214 & 91 & 123 \\
\hline & 9 & 3.0 & 92 & 75 & 17 & 217 & 102 & 115 \\
\hline \multirow{2}{*}{100} & 2 & 3.1 & 79 & 80 & -1 & 183 & 81 & 102 \\
\hline & 4 & 3.2 & 90 & 74 & 16 & 243 & 97 & 146 \\
\hline \multirow{2}{*}{150} & 17 & 3.7 & 109 & 84 & 25 & 213 & 94 & 119 \\
\hline & 18 & 3.9 & 91 & 77 & 14 & 306 & 106 & 200 \\
\hline \multirow{2}{*}{200} & 1 & 3.2 & 86 & 95 & -9 & 109 & 75 & 34 \\
\hline & 7 & 2.8 & 102 & 85 & 17 & 158 & 83 & 75 \\
\hline \multirow{2}{*}{250} & 16 & 4.0 & 82 & 81 & 1 & 243 & 91 & 152 \\
\hline & 19 & 2.6 & 91 & 83 & 8 & 234 & 103 & 131 \\
\hline
\end{tabular}

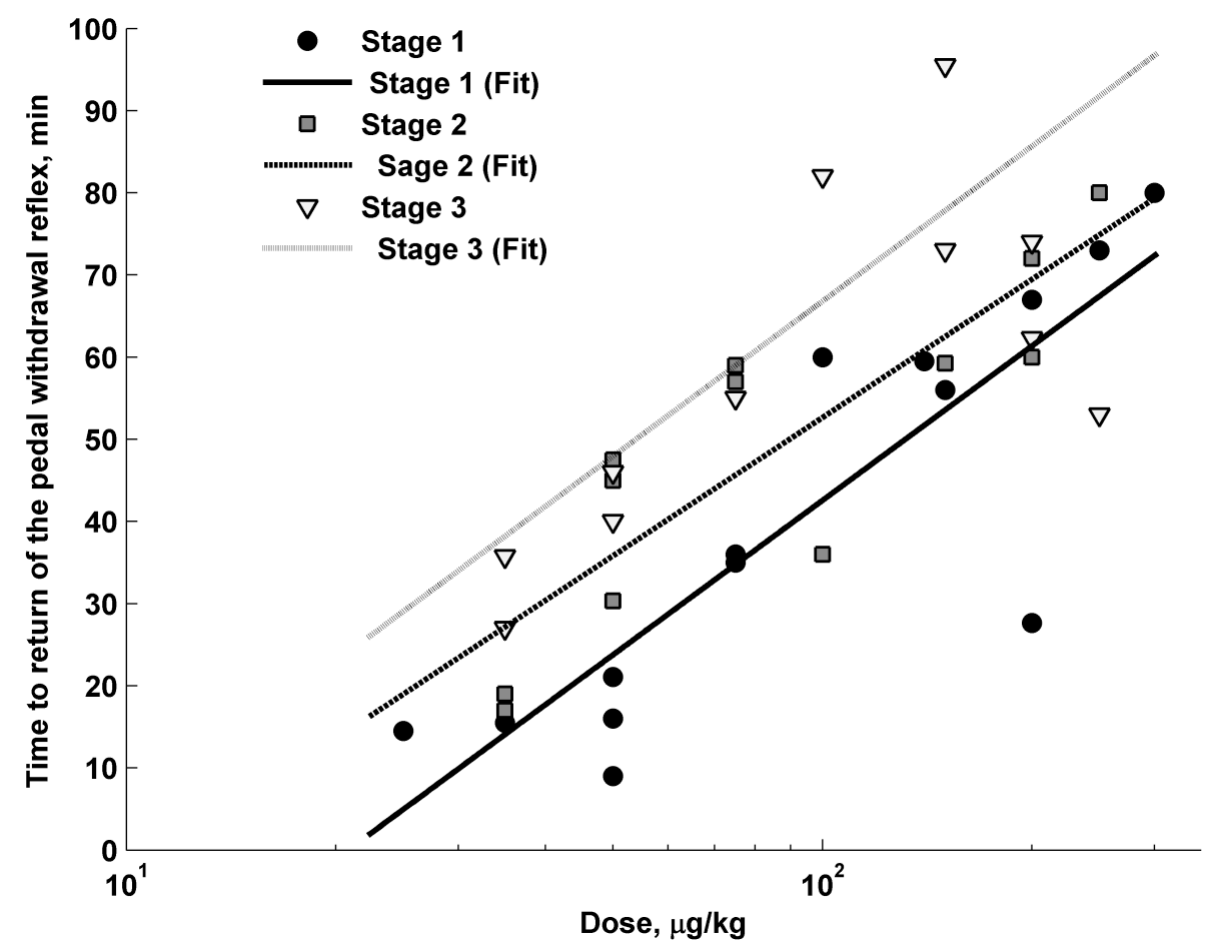

Figure 1. The relationship between the dexmedetomidine dose and the time to return of the pedal withdrawal reflex. The symbols denote the experimental data. The straight lines indicate the best fit of the Eq. 1 to the data for each stage

and young rabbits. There have been numerous studies that used this reflex to examine other drugs, such as propofol, midazolam, pentobarbital alone or in mixtures: diazepam-ketamine-pentazocine, katamine-xylazine, midazolam-xylazine-alfentanil [11, 12, 13, 21]. Our study was unique for the following reasons. First of all, we divided the study into 3 periods, taking into account the process of maturation of all animals individually. At stage 1 we chose young rabbits ( $42-54$ days old), mostly because there is no available literature on intravenous administration of dexmedetomidine to this age group. We conducted the consecutive stages of the study with the same animals but at different ages to determine the influence of their maturation process on the pharma- 

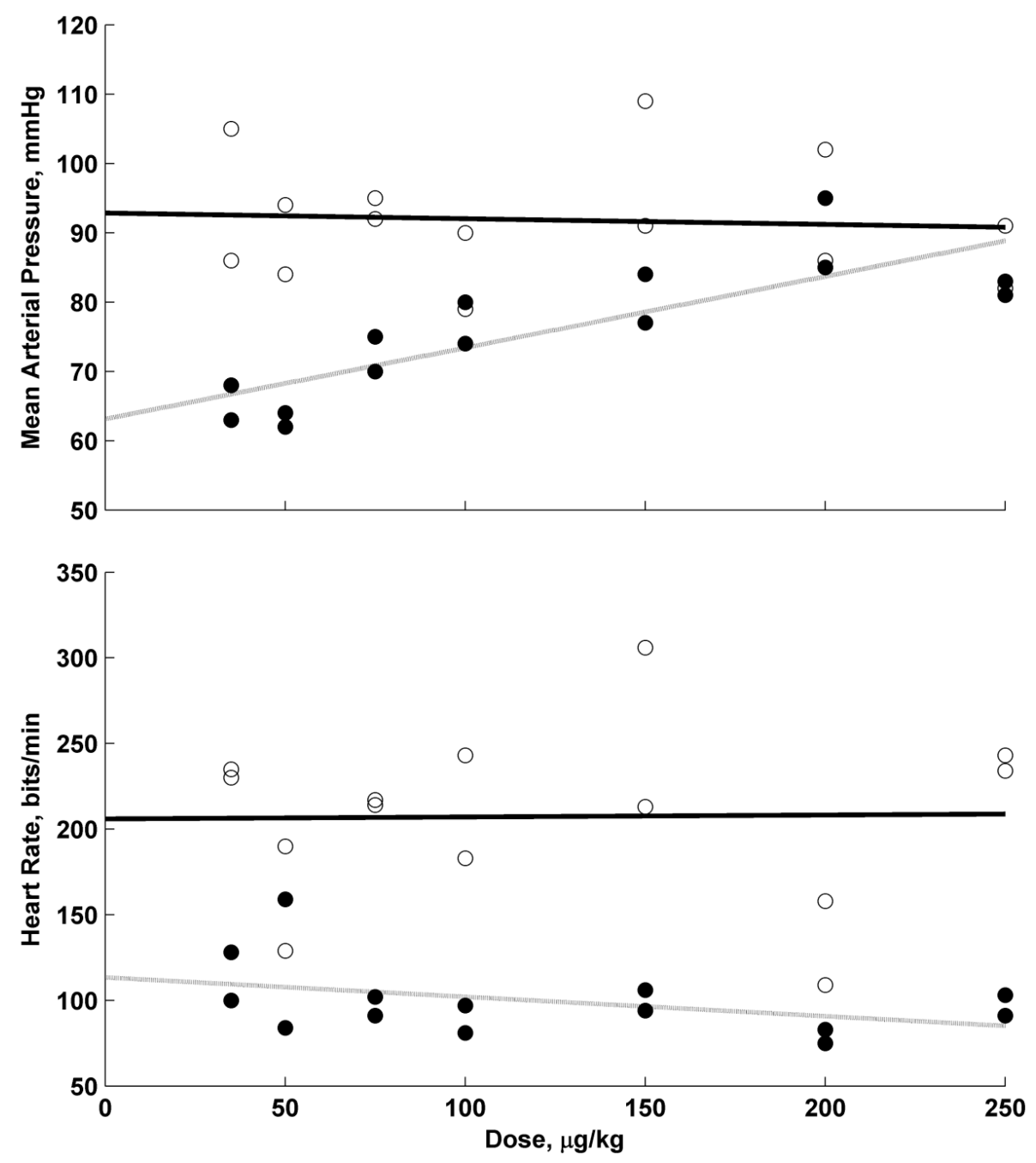

Figure 2. The relationship between the dexmedetomidine dose and mean arterial pressure and heart rate. The open symbols denote the baseline values and closed symbols the mean values noted during the anaesthesia for each rabbit. The straight lines indicate the trends in the data

codynamics of dexmedetomidine. Thus, we eliminated the problem of interindividual variability. We chose the rabbits whose weight was around $1 \mathrm{~kg}$, as the titration of dexmedetomidine in smaller animals is more difficult and thus its application is limited [22]. For the first time we also examined the linearity of the pharmacodynamics of dexmedetomidine and its haemodynamic effects on rabbits at very narrow dosage intervals. The linearity of the pharmacokinetics of dexmedetomidine is of great clinical importance because there is only a limited number of data concerning the pharmacokinetics of this drug administered in prolonged ( $>24 \mathrm{~h}$ ) infusion at large doses (up to $2.5 \mu \mathrm{g} / \mathrm{kg} / \mathrm{h}$ ) and there are no such studies on children [23]. The results show the linear relationship between the logarithm of the administered dose of dexmedetomidine and the duration of sedation measured with the pedal withdrawal reflex. During our study we observed that as the rabbits grew older, they became more sensitive to the sedative effects of dexmedetomidine. We cannot transfer the results of our studies directly to humans, but we can conclude that children may require higher doses of dexmedetomidine than adults. However, it is necessary to make a pharmacokinetic analysis to explain this phenomenon fully and we are planning to do it in further parts of our project. Moreover, in the third period we examined the influence of different doses of the drug on the haemodynamic response. Thus, we confirmed the hypotonic effect of dexmedetomidine. As far as this effect is concerned, we noted that in spite of the fact that dexmedetomidine is known for its hypotensive effect, as doses of the drug increased, the blood pressure tended to increase, too. Potts et al. conducted a study on children after a cardiac surgery. The aim of their research was to determine the influence of the pharmacodynamics of dexmedetomidine on the haemodynamics of the circulatory system 
in the children who had undergone cardiac surgeries and received a bolus dose of $1-4 \mu \mathrm{g} / \mathrm{kg}$ within $10 \mathrm{~min}$ utes. Large doses of dexmedetomidine were observed to cause the contraction of smooth muscles in blood vessels. This resulted in a short increase in the arterial pressure, whereas the application of small doses of the drug had the hypotensive effect [24]. Three doses of dexmedetomidine were previously applied to adult rabbits $(20,80$ or $320 \mu \mathrm{g} / \mathrm{kg})$ by Zornow who studied the haemodynamic effect of dexmedetomidine on rabbits. He noted that the administration of the three doses under study caused a decrease in the heart rate in a significant and dosage-related fashion, whereas the mean arterial blood pressure did not change significantly at any dose or time. Similarly to Zornow, we noted a significant decrease in the heart rate. However, contrary to his findings, we observed significant dosage-related changes in the MAP. In our study we observed that small doses of dexmedetomidne resulted in a significant drop in the blood pressure, but this effect was less noticeable with higher doses of the drug. The most important difference between these two studies consists in the fact that although our doses were the same as Zornow's, the intervals were more naoorw $(25,35,50,75,100,140$, $150,200,250,300 \mu \mathrm{g} / \mathrm{kg}$ ) [20].

To conclude, young rabbits are less sensitive to the sedative pharmacodynamic response of dexmedetomidine than adult animals. The haemodynamic response to dexmedetomidine depends on dosage of the drug. There was a significant decrease in the blood pressure observed with small doses of the drug, but the effect was less noticeable with higher doses. After the administration of dexmedetomidine the heart rate decreased regardless of the dose applied.

\section{Conflict of interest}

The authors declare that there are no conflicts of interest.

$$
\begin{aligned}
& \text { Abbreviations } \\
& \text { ICU - Intensive Care Unit } \\
& \text { GABA - Gamma Aminobutyric Acid } \\
& \text { PICU - Pediatric Intensive Care Unit } \\
& \text { PK - Pharmacokinetics } \\
& \text { PD - Pharmacodynamic } \\
& \text { MAP - mean oriented blood pressure }
\end{aligned}
$$

\section{References}

1. Afshani N. Clinical application of dexmedetomidine. S Afr J Anaesthesiol Analg. 2010;16(3):50-6.

2. Jones CR. Perioperative uses of dexmedetomidine. Int Anesthesiol Clin. 2013;51(2):81-96.
3. Maldonado JR, Wysong A, van der Starre PJ, Block T, Miller $C$, Reitz BA. Dexmedetomidine and the reduction of postoperative delirium after cardiac surgery. Psychosomatics. 2009;50(3):206-17.

4. Holliday SF, Kane-Gill SL, Empey PE, Buckley MS, Smithburger PL. Interpatient variability in dexmedetomidine response: a survey of the literature. Scientific World Journal. 2014 Jan 16;2014:805013.

5. Tobias JD. Dexmedetomidine: applications in pediatric critical care and pediatric anesthesiology. Pediatr Crit Care Med. 2007;8(2):115-31.

6. De Cock RF, Piana C, Krekels EH, Danhof M, Allegaert K, Knibbe CA. The role of population PK-PD modelling in paediatric clinical research. Eur J Clin Pharmacol. 2011;67 Suppl 1:5-16.

7. Funk RS, Brown JT, Abdel-Rahman SM. Pediatric pharmacokinetics: human development and drug disposition. Pediatr Clin North Am. 2012;59(5):1001-16.

8. Petroz GC, Sikich N, James M, van Dyk H, Shafer SL, Schily M, Lerman J. A phase I Two-center Study of the Pharmacokinetics and Pharmacodynamics of Dexmedetomidine in Children. Anesthesiology. 2006;105:1098-110.

9. The European Regulation on medicines for paediatric use. Paediatr Respir Rev. 8(2):177-83.

10. Consultant PR (2006) EU paediatric regulation for testing medicines in children. http://www.child-medicines-research-info.com/paediatric-research-consultancy/eu-paediatric-regulation.asp. 23 June 2009.

11. Bienert A, Płotek W, Zawidzka I, Ratajczak N, Szczesny D, Wiczling P, et al. Influence of time of day on propofol pharmacokinetics and pharmacodynamics in rabbits. Chronobiol Int. 2011;28(4):318-29.

12. Bienert A, Płotek W, Wiczling P, Kostrzewski B, Kamińska $A$, Billert $\mathrm{H}$, et al. The influence of the time of day on midazolam pharmacokinetics and pharmacodynamics in rabbits. Pharmacological Reports. 2014;66(1):143-52.

13. Adetunji A, Oguntoye CO, Esho VO. Evaluation of diazepam-ketamine-pentazocineanaesthesia in rabbits. Afr J Biomed Res. 2009;12(3):237-40.

14. Gibaldi M, Levy G. Dose-dependent decline of pharmacologic effects of drugs with linear pharmacokinetic characteristics, J Pharm Sci. 1972;61(4):567-9.

15. Bol CJ, Danhof M, Stanski DR, Mandema JW. Pharmacokinetic-pharmacodynamic characterization of the cardiovascular, hypnotic, EEG and ventilatory responses to dexmedetomidine in the rat. J Pharmacol Exp Ther. 1997;283(3):1051-8.

16. Kuusela E, Raekallio M, Anttila M, Falck I, Mölsä S, Vainio O. Clinical effects and pharmacokinetics of medetomidine and its enantiomers in dogs. J Vet Pharmacol Ther. 2000;23(1):15-20.

17. Ansah OB, Raekallio M, Vainio O. Comparison of three doses of dexmedetomidine with medetomidine in cats following intramuscular administration. J Vet Pharmacol Ther. 1998;21(5):380-7.

18. Kuhmonen J, Pokorný J, Miettinen R, Haapalinna A, Jolkkonen J, Riekkinen P Sr. Neuroprotective effects of dexmedetomidine in the gerbil hippocampus after transient global ischemia. Anesthesiology. 1997;87(2):371-7.

19. Cosar M1, Eser O, Fidan H, Sahin O, Buyukbas S, Ela Y. The neuroprotective effect of dexmedetomidine in the hippocampus of rabbits after subarachnoid hemorrhage. Surg Neurol. 2009;71(1):54-9. 
20. Zornow MH. Ventilatory, hemodynamic and sedative effects of the alpha 2 adrenergic agonist, dexmedetomidine. Neuropharmacology. 1991;30(10):1065-71.

21. Borkowski GL, Danneman PJ, Russell GB, Lang CM. An evaluation of three intravenous anesthetic regimens in New Zealand rabbits. Lab Anim Sci. 1990;40(3):270-6.

22. Varga M. Textbook of Rabbit Medicine. $2^{\text {nd }}$ ed. BH Elsevier, Oxford 2003, 187.

23. lirola T, Aantaa R, Laitio R, Kentala E, Lahtinen M, Wighton $A$, et al. Pharmacokinetics of prolonged infusion of high-dose dexmedetomidine in critically ill patients. Crit Care. 2011;15(5):R257.
24. Potts AL, Anderson BJ, Holford NH, Vu TC, Warman GR. Dexmedetomidine hemodynamics in children after cardiac surgery. Paediatr Anaesth. 2010;20(5):425-33.

Correspondence address: Agnieszka Bienert

Department of Clinical Pharmacy and Biopharmacy Poznan University of Medical Sciences 14 Św. Marii Magdaleny Street 61-861 Poznań, Poland email: agnbienert@op.pl 